OPEN ACCESS

Edited by:

Jian Yu,

Beihang University, China

Reviewed by:

Hongyang Li,

University of Michigan, United States

Fu Gui,

Brigham and Women's Hospital and Harvard Medical School, United States

*Correspondence:

Feng Wang

wfeng@bit.edu.cn

Specialty section:

This article was submitted to Cancer Metabolism,

a section of the journal

Frontiers in Oncology

Received: 13 December 2021 Accepted: 03 January 2022 Published: 26 January 2022

Citation:

Lan H, Gao Y, Zhao Z, Mei Z and Wang $F$ (2022) Ferroptosis:

Redox Imbalance and Hematological Tumorigenesis.

Front. Oncol. 12:834681. doi: 10.3389/fonc.2022.834681

\section{Ferroptosis: Redox Imbalance and Hematological Tumorigenesis}

\author{
Hongying Lan ${ }^{1}$, Yu Gao ${ }^{1}$, Zhengyang Zhao ${ }^{1}$, Ziqing $\mathrm{Mei}^{2}$ and Feng Wang ${ }^{1 *}$ \\ ${ }^{1}$ Key Laboratory of Molecular Medicine and Biotherapy, School of Life Science, Beijing Institute of Technology, Beijing, \\ China, 2 School of Chemistry and Biological Engineering, University of Science and Technology Beijing, Beijing, China
}

Ferroptosis is a novel characterized form of cell death featured with iron-dependent lipid peroxidation, which is distinct from any known programmed cell death in the biological processes and morphological characteristics. Recent evidence points out that ferroptosis is correlated with numerous metabolic pathways, including iron homeostasis, lipid metabolism, and redox homeostasis, associating with the occurrence and treatment of hematological malignancies, such as multiple myeloma, leukemia, and lymphoma. Nowadays, utilizing ferroptosis as the target to prevent and treat hematological malignancies has become an active and challenging topic of research, and the regulatory network and physiological function of ferroptosis also need to be further elucidated. This review will summarize the recent progress in the molecular regulation of ferroptosis and the physiological roles and therapeutic potential of ferroptosis as the target in hematological malignancies.

Keywords: ferroptosis, redox, hematologic malignancies, lipid metabolism, iron homeostasis

\section{INTRODUCTION}

Death is an irreversible regulation process in the living cells, and different ways of death relate to the distinct physiological functions. Cell death is divided into programmed cell death and cell necrosis. Programmed cell death is an actively induced and tightly controlled process of cell suicide in response to various signal stimuli. In contrast, cell necrosis is an acute, spontaneous and passive death caused by unrepairable stress under pathological conditions, such as physical, chemical, hypoxia, or insufficient energy (mainly ATP) (1-6). When the cell is necrotic, the integrity of the cytoplasmic membrane is destroyed, and the barrier function to $\mathrm{Na}^{+}, \mathrm{Ca}^{2+}$, and water will be lost. Water flowing into the cell could lead to cytoplasmic swelling and nucleus pyknosis, eventually leading to cell rupture (7). The most canonical way of programmed cell death is apoptosis, which relates to cell contraction, chromatin condensation, and the formation of apoptotic bodies. With the continuous advancement of research on the manner of cell death, it has been found that in addition to apoptosis, programmed cell death also comprises autophagy, programmed necrosis, pyroptosis, and ferroptosis. Ferroptosis is a new form of cell death discovered by Stockwell in searching for small molecules targeted at RAS protein mutations related to cancer. Its morphological characteristics are different from any known form of cell death, as shown in Figure 1 (8). Irondependent cell membrane lipid peroxidation will lead to ferroptosis. At this time, the cell's mitochondrial membrane density will increase, while the mitochondrial cristae will decrease or disappear, and the mitochondrial outer membrane will rupture, but the nucleus will remain normal $(9,10)$. Since ferroptosis was defined as a new form of cell death in 2012 , more and more researchers 

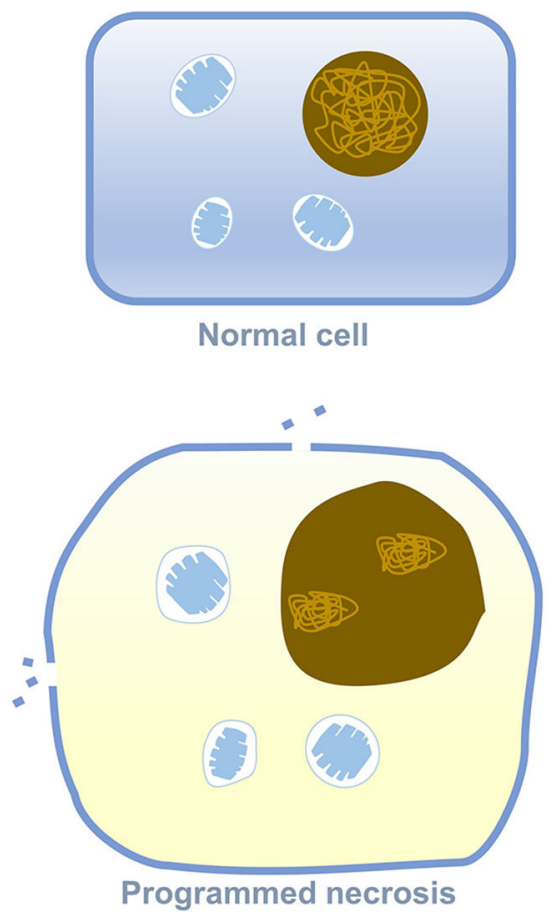

Mitochondria

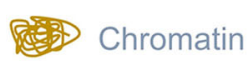

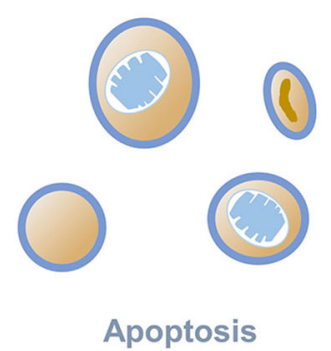

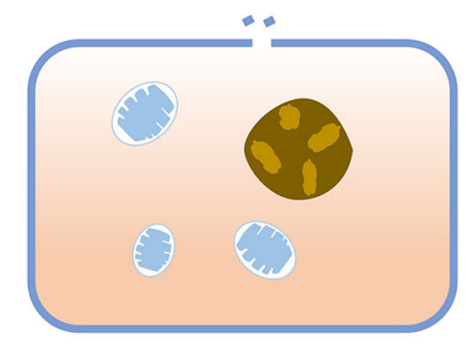

Pyroptosis
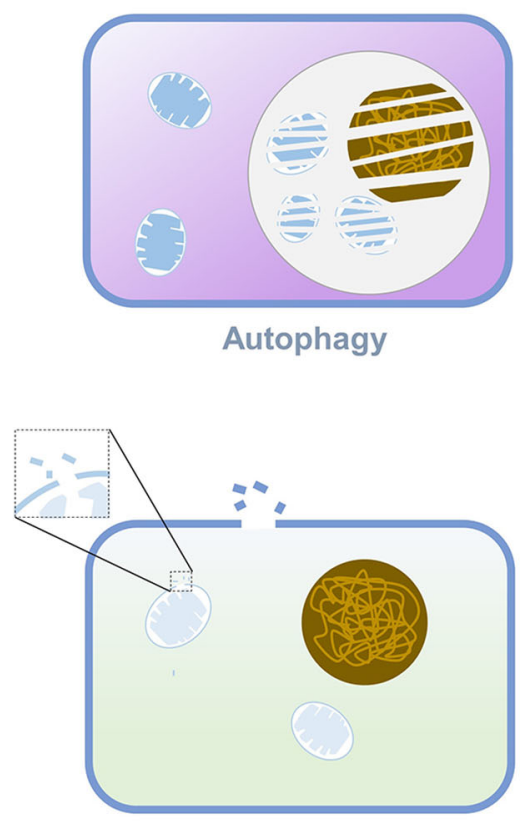

Ferroptosis

Autolysosome

FIGURE 1 | Morphological Features of different programmed cell death.

have garnered significant attention on ferroptosis and continuously identified the correlation of ferroptosis with cancer and tumor immunity. This review will summarize recent progress on the regulatory mechanism of ferroptosis and the pathological manifestations related to ferroptosis and propose potential treatment strategies.

\section{MECHANISM OF FERROPTOSIS}

\section{Lipid Peroxidation Leads to Ferroptosis}

The most apparent feature of ferroptosis in cells is lipid peroxidation. In the process of ferroptosis, the initial and essential step is that ACSL4, as a member of the acyl-CoA synthase long-chain family (ACSL) family, specifically catalyzes polyunsaturated fatty acid (PUFA), such as arachidonoyl (AA) and adrenoyl moieties (AdA), to form a long-chain acyl-CoA, namely PUFA-CoA (11). Downregulation of ACSL4 expression or pharmacological inhibition of ACSL 4 activity (thiazolidinediones or triacsin $\mathrm{C}$, etc.) can prevent ferroptosis (12-14). Lysophosphatidylcholine acyltransferase 3 (LPCTA3) then selectively uses phosphatidylethanolamine (PE) or phosphatidylcholine (PC) located on the endoplasmic reticulum as the receptor for acylated PUFA to generate
PUFA-PE or PUFA-PC (15). Lipids with unsaturated fatty acids are typical peroxidation targets because carbon-carbon double bonds are susceptible to reactive oxygen species (ROS). According to different peroxidation mechanisms, lipid peroxidation can be divided into enzymatic and non-enzymatic types. It is currently considered that the enzyme pathway is mainly accomplished by lipoxygenase (LOX), which is a class of dioxygenases containing non-heme iron and can directly catalyze the peroxidation of PUFA-PE $(13,16,17)$. Six LOX species have been identified in the human genome and referred to as 5-LOX, 12-LOX, 15-LOX-1, 15-LOX-2, 12R-LOX, and eLOX3 according to their oxidation positions on the arachidonic acid carbon chain $(11,18)$. When LOX is overexpressed, cells appear to be sensitive to ferroptosis, whereas inhibiting LOX activity, in turn, protects cells from RSL3-induced ferroptosis (19). The enzymatic lipid peroxidation is the reaction where enzymes specially select and catalyze substrates to generate the products. In non-enzymatic lipid peroxidation, free and unstable ferrous ions react with hydrogen peroxide to generate ferric ions and strongly oxidizing hydroxyl radicals $(\mathrm{OH}$.). Hydroxyl radicals abstract the first hydrogen in the PUFA and form resonantly stable carbon-centered lipid radical (PUFA-R.), which can react with molecular oxygen to form lipid hydroperoxyl radical (PUFAROO.). Another hydrogen can be extracted by lipid peroxidation 
radical from the adjacent unsaturated fat chain, leading to the formation of lipid peroxides (PUFA-ROOH) and new resonant carbon center radicals. In this cycle, the chain reaction continues to proceed and generates new lipid peroxides until the concentration of PUFA-ROO is high enough for two PUFAROO to contact each other so that a new bond is formed (20-22). Lipid peroxidation can generate unstable hydroperoxyl groups in PUFA and promote oxidative truncation of PUFA-ROOH, creating electrophiles such as aldehydes and Michael receptors. The electrophilic products then attack proteins on the cell membrane, causing plasma membrane rupture and cell death $(23,24)$.

As a natural fat-soluble antioxidant, $\alpha$-tocopherol can disrupt the chain reaction in lipid peroxidation and inhibit ferroptosis, owing to its high affinity for unpaired electrons $(25,26)$. Liproxstatin-1 and ferrostatin-1, two ferroptosis inhibitors identified by high throughput screening, have the characteristics of free radical-trapping antioxidants (RTA), and hence preventing ferroptosis by scavenging free radicals $(8,27$, 28). Recent studies have demonstrated that cytochrome P450 oxidoreductase (POR) and cytochrome B5 reductase 1 (CYB5R1) can transfer electrons from $\mathrm{NAD}(\mathrm{P}) \mathrm{H}$ to downstream proteins such as cytochrome $\mathrm{P} 450$ (CYP), which incorrectly transfer electrons to molecular oxygen to generate hydrogen peroxide. The Fenton reaction between hydrogen peroxide and ferrous ions can induce ferroptosis (29). When intracellular expression of POR or CYB5R1 was downregulated, the $\mathrm{H}_{2} \mathrm{O}_{2}$ content was reduced, while the cell survival rate was remarkably increased $(29,30)$.

\section{Lipid Peroxidation Defense System}

As lipid supports the structure of the cell membrane or organelle membrane, lipid peroxidation can significantly change the physical properties of the lipid bilayer. As a marker and necessary prerequisite of ferroptosis, the accumulation of lipid peroxidation is regulated by various redox systems in cells (Figure 2) (21).

\section{GPX4-GSH System}

As a negative regulator for ferroptosis, GPX4 utilizes two reduced glutathione molecules ( $\gamma$-glutamyl-cysteinyl-glycine, GSH) as the electron donor to reduce lipid peroxides (such as $\mathrm{AA}-\mathrm{OOH})$ into corresponding alcohols $(\mathrm{AA}-\mathrm{OH})$ and produce a molecule of oxidized glutathione (GSSG), reducing lipid peroxidation and preventing ferroptosis $(31,32)$.

The production of GSH in cells is inseparable from the system Xc- a heterodimer composed of SLC3A2 and SLC7A11, which can execute the antiport of cystine and glutamate on the cell membrane, namely takes one molecule of cystine into the cell and releases one molecule of glutamate from the cell (33). Cystine transported into the cell is rapidly reduced to cysteine, which is involved in GSH synthesis and other physiological reactions. The $\gamma$-glutamylcysteine synthase $(\gamma$-GCS) performs the first and rate-limiting step in the process of GSH synthesis: synthesis of L- $\gamma$-glutamylcysteine from L-cysteine and Lglutamate in the presence of ATP, while GSH synthase catalyzes the synthesis of GSH from glycine and $\gamma$ glutamylcysteine $(34,35)$. After erastin treatment, ferroptosisinducing agents56 (FIN56) can inhibit GPX4 and cells exhibit susceptibility to ferroptosis. When GPX4 is overexpressed, FIN56-induced ferroptosis would be impeded (36). Similarly, dihydroartemisinin (DHA) can also promote ferroptosis in glioblastoma through targeted downregulation of GPX4 and accumulation of lipid peroxidation (37). Albeit GPX4-deficient hematopoietic stem cells are prone to suffer from ferroptosis in vitro, GPX4-deficient mice still retain general function, owing to the antioxidant function of lipophilic vitamin E. Moreover, GPX4 combined with vitamin E can also prevent hepatocellular disease $(38,39)$.

\section{FSP1-CoQ 10 System}

FSP1-CoQ 10 system can antagonize ferroptosis in GPX4-GSH system-independent mechanism. Doll and Bersuker's teams almost simultaneously demonstrated that FSP1 was strongly associated with ferroptosis $(40,41)$. FSP1 mainly contains two distinct structural domains, the N-terminal short hydrophobic region and $\mathrm{FAD}$-dependent $\mathrm{NAD}(\mathrm{P}) \mathrm{H}$ redox region, and participates in ferroptosis through reduction of ubiquinone (also known as coenzyme $\mathrm{Q}_{10}, \mathrm{CoQ}_{10}$ ) and formation of panthenol $\left(\mathrm{CoQ}_{10} \mathrm{H}_{2}\right)$, which is a reduced form of the fatsoluble antioxidant ubiquinone and can trap and reduce lipid peroxidation radicals (42). In addition, the overexpression of FSP1 in most tumor cells, and the treatment with the inhibitor of FSP1 (iFSP1), can make cells sensitive to RSL3 (43). Meanwhile, Bersuker et al. identified that FSP1 was a negative regulator of ferroptosis in screening for the synthesis of lethal CRISPR-Cas9, and the localization of FSP1 to the plasma membrane by myristoylation was necessary for its inhibition of ferroptosis (31). Subsequent studies demonstrated that FSP1 plays a role in membrane repair and resistance to ferroptosis in the panthenol-independent and ESCRT-III-dependent manner (44). Knockdown of FSP1 inhibits the expression of ESCRT-III subunits (CHMP5 and CHMP6), but the exact mechanism remains unclear. Furthermore, mice with knockout of the FSP1 gene did not show any abnormalities before at least one-year-old (45). In conclusion, promoting ferroptosis in tumor cells via targeting inhibition of FSP1would be a potential strategy for cancer therapy. Consistently, the development of FSP1 inhibitors is also of great significance for clinical research.

\section{GCH1-BH4 System}

Human GCH1 is a $270 \mathrm{kD}$ complex composed of five dimers that catalyzes the conversion of guanosine triphosphate (GTP) to dihydroneopterin triphosphate (H2NTP), which is the first and rate-limiting step in the biosynthesis of $\mathrm{BH} 4$ (46). H2NTP was then transformed into $\mathrm{BH} 4$ by 6-pyruvoyl tetrahydrobiopterin synthase (PTS) and sepiapterin reductase (SPR). Kraft found that GCH1 was related to ferroptosis through CRISPR-Cas9 overexpression screening. GCH1 prevents the peroxidation of phosphatidylcholine with two polyunsaturated fatty acid chains to prevent ferroptosis through the antioxidant action of $\mathrm{BH} 4$, and $\mathrm{BH} 4$ may also be involved in the pathway of FSP1-CoQ 10 as a biosynthetic precursor of $\mathrm{CoQ}_{10}$ (47). Dihydrofolate reductase 


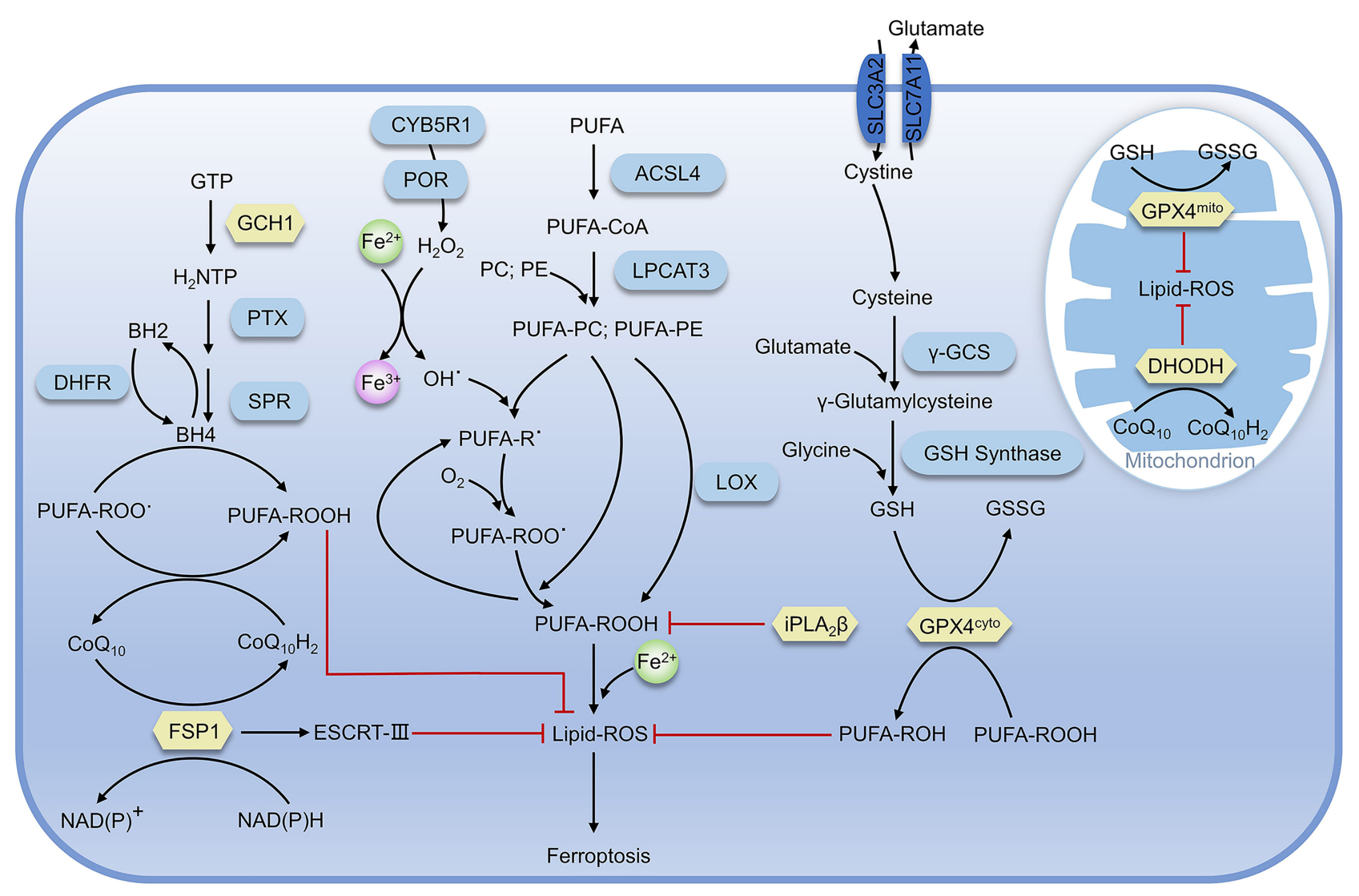

FIGURE 2 | Regulatory pathways of ferroptosis. The proteins in yellow are key defense systems; the red line represents inhibiting effect (as in Figure 4).

(DHFR) participates in the regulation of ferroptosis by regenerating oxidized $\mathrm{BH} 4$ and the combination of inhibition of DHFR by methotrexate (MTX) with inhibition of GPX4 by RSL3 can promote ferroptosis $(48,49)$. In summary, BH4 is the core element of this system for ferroptosis resistance. In addition to GCH1 and DHFR, there are other factors mediating ferroptosis through $\mathrm{BH} 4$, and the imbalance of $\mathrm{BH} 4$ levels may be associated with the occurrence of ferroptosis-related diseases.

iPLA $_{2} \beta$

$\mathrm{Ca}^{2+}$-independent phospholipase $\mathrm{A}_{2} \beta$ ( $\mathrm{iPLA}_{2} \beta$ ) can specifically hydrolyze sn-2 acyl bonds of phospholipids, which has been recently identified as a regulator of ferroptosis. When ferroptosis occurs, the characteristic product of lipid peroxidation, 15hydroperoxy-arachidonoyl-phosphatidylethanolamine (15HpETE-PE), is hydrolyzed by $\operatorname{iPLA}_{2} \beta$, which impedes subsequent ferroptosis-related effects. Decreased iPLA ${ }_{2} \beta$ expression in cells was significantly more sensitive to RSL3induced ferroptosis and showed higher ferroptosis markers associated with PE (50). The interaction of ${ }^{2} \mathrm{PLA}_{2} \beta$ with various membrane substrates was simulated by computational modeling, and it was found that 15-HpETE-PE was more exposed to the membrane surface, close to the catalytic site (50). When the R747W mutation occurred in the catalytic domain, the interaction with the membrane was diminished, leading to a decline in the catalytic capacity of 15-HpETE-PE and the inhibition of ferroptosis in cells. It is demonstrated that the reduced enzyme activity may be linked to neurological diseases, including Parkinson's disease in particular. Besides, iPLA2 $\beta$ was further identified as a regulator of p53-mediated ferroptosis, independent of GPX4 and FSP1, inhibiting ferroptosis by eliminating AlOX12-catalyzed lipid peroxidation. Notably, like FSP1, the lack of iPLA2 $\beta$ has no impact on cell function or tissue development, suggesting that it may be a potential target for inducing ferroptosis in tumor cells (51).

\section{DHODH}

Mitochondria is the indispensable organelle in eukaryotes where oxidative phosphorylation, energy generation, and important other functions such as signal transmission and energy metabolism occur. Mao et al. recently demonstrated that dihydroorotate dehydrogenase (DHODH) mediated the regeneration of panthenol in mitochondria to restore peroxidedamaged mitochondrial lipids and inhibition of ferroptosis by DHODH was independent of DHODH's function in pyrimidine synthesis (52). DHODH can also produce panthenol to repair peroxide lipids, but DHODH targets mitochondrial membrane lipids instead of cytoplasmic lipids. GPX4 is subdivided into 
cytoplasmic and mitochondrial types, which are referred to as GPX $4^{\text {cyto }}$ and GPX $4^{\text {mito }}$, respectively. GPX $4^{\text {mito }}$ and DHODH protect the mitochondria against oxidative damage independent of GPX $4^{\text {cyto }}$ and FSP1. Mao also demonstrated that Brequinar, an inhibitor of DHODH, impeded the proliferation of tumor cells with low GPX4 expression. In addition, combined use of ferroptosis inducers could inhibit the growth of tumor cells with high GPX4 expression, implying a new approach for cancer treatment (52).

\section{METABOLIC REGULATION OF FE ${ }^{2+}$}

Iron is a vital transition metal with redox activity in the body, which has implications in biological processes such as oxidative phosphorylation, DNA synthesis, and cell signaling $(53,54)$. Excessive or inadequate iron levels can lead to the loss of protein function, abnormalities in intracellular signaling, as well as outof-control of metabolic networks, thus interfering with normal physiological processes (55). Ferrous ions are reported to be involved in the Fenton reaction and promote lipid peroxidation when ferroptosis occurs. In addition, reactive oxygen species (ROS) that trigger the Fenton reaction are also related to iron (56).

In a natural evolution, organisms have evolved multiple regulatory pathways of iron homeostasis. Most of the ferric ions in nutrients are absorbed by the brush border of duodenal cells, reduced to ferrous ions by duodenal cytochrome b (Dcytb), then transported to intestinal cells by Divalent metal ion Transporter 1 (DMT1) (57). In intestinal cells, a part of ferrous ion is fixed by ferritin, while the other parts are utilized by the ferroportin (FPN, also known as SLC40A1) and transported into the blood. Ferrous ions are oxidized to ferric ions in the blood by Hephaestin (HEPH) or Ceruloplasmin (CP) $(53,58)$. Transferrin (Tf) can bind two ferric ions and enter into cells through endocytosis after forming a complex with transferrin receptor (TfR). In the acidic environment of the Endosome, ferric ions are dissociated from Tf and reduced to ferrous ions by a sixtransmembrane epithelial antigen of the prostate 3 (STEAP3), and then are transported to the cytoplasm by DMT1, while Tf and TfR can be recycled for the next transfer $(57,59,60)$. Ferric ions entering the cytoplasm can function in the various physiological processes or stay in ferritin.

Increased TfR expression was found in erastin-induced ferroptosis and p53 induced ferroptosis, suggesting that TfR is positively associated with ferroptosis $(61,62)$. In addition, it has been found that knockdown of FPN in neuroblastoma promotes ROS-dependent ferroptosis, and the downregulation of FPN expression leads to ferroptosis in the hippocampal area of rats and other diabetic cognitive dysfunction $(63,64)$. The storage of iron ions in cells is undertaken by ferritin, which is composed of a heavy chain (FTH) and a light chain (FTL) and forms a "labile iron pool (LIP)" of 12 or 24 polymers through the weak interaction. The storage pool can store more than 4500 ferrous ions. Ferritins from different species are distinct in ferritin size, amino acid sequence, iron access channel, and iron-binding site.
However, from the aspects of shape and structure, they are all "pools" formed by orderly arranged helixes $(65,66)$. The iron storage pools control the concentration of free ferrous ions in the cytoplasm, which determines whether it acts as a beneficial cofactor or as a toxic-free radical catalyst in the cell and is key for the fate of the cell (Figure 3).

Nuclear Receptor Coactivator-4 (NCOA4) mediates the degradation of ferritin in an autophagy-dependent pathway where iron is isolated from ferritin and the accumulated free ferrous ions in the cytoplasm promote erastin-induced ferroptosis (67). Nuclear factor-erythroid 2-related factor 2 (NRF2) regulates the expression of FPN1, FTH, and FTL at the transcriptional level and promotes the stability of the intracellular iron environment (68). TfR and ferritin translation regulation at the transcriptional level in a cell operates through IRE-IRP. Iron regulatory proteins (IRPs) can be classified into IRP1 and IRP2. IRP1 can be converted between apo-form and holo-form, wherein the former can bind mRNA, and the latter has aconitase activity and is the main active form of IRP1 $(69,70)$. IRP2 is widespread in mammals and is the main executor of IRE-IRP iron regulation $(69,71)$. When the intracellular iron concentration is inadequate, IRP will bind to the 3' untranslated regions of mRNA (UTR) of TfR with a high affinity and specific manner to enhance the stability of the mRNA and the expression level of TfR, resulting in increased iron transport into the cell (72). When intracellular iron is abundant, IRP binds to the 5' UTR of ferritin and TfR, preventing their translation. IRP is at the control of intracellular iron level and $\mathrm{H}_{2} \mathrm{O}_{2}$, oxygen concentration, and oxidative stress signal $(72,73)$.

\section{REGULATORY MECHANISMS ASSOCIATED WITH FERROPTOSIS}

\section{Effect of Ether Phospholipid on Ferroptosis}

Unlike typical fatty acids, which are connected to the glycerol framework via two ester bonds, the sn-1 position of ether phospholipids (ePLs) is linked to the aliphatic chain via the ether bond, while the position of sn-2 is linked to the polyunsaturated fat chain by an ester bond (74, 75). Carbons adjacent to ether bonds can be bonded in two ways: the first is a carbon-carbon single bond to form an alkyl ether; another is a carbon-carbon double bond for the formation of vinyl ethers (also known as acetal phospholipids). Zou et al. found that peroxisome composition was associated with ferroptosis using genome-wide CRISPR-Cas9 suppressor screens, in combination with the protein network database STRING and the pathway analysis algorithm they developed. Furthermore, they found utilizing lipidomics that polyunsaturated ether phospholipids (ePLs) generated by the peroxisome pathway act as substrates of lipid peroxidation to induce ferroptosis (76). When the expression of ether phospholipid-related synthases Alkyldihydroxyacetonephosphate synthase (AGPS) and Fatty acyl-CoA reductase 1 (FAR1) in peroxisome was inhibited, the 


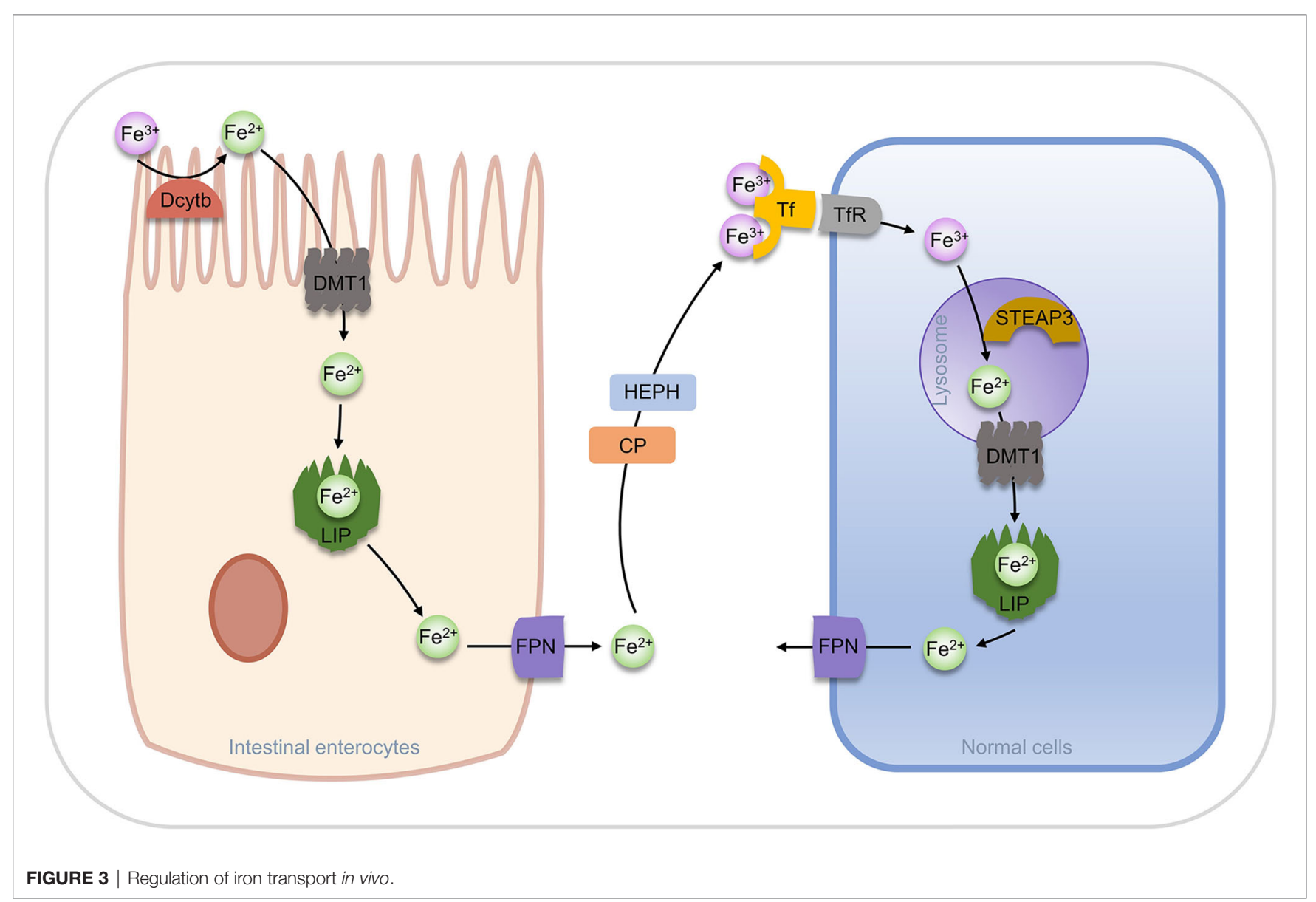

cells would exhibit the resistance to ferroptosis until they were re-expressed. In another study, Cui et al. established the regulatory pathway of ferroptosis involving FAR1 as FAR1ether lipid-TMEM189 (77). FAR1 promotes ferroptosis by reducing fatty acids to generate fatty alcohols necessary for synthesizing alkyl ether lipids and acetal phospholipids (77). Cui found that acetal phospholipids generated by plasmanylethanolamine desaturase (transmembrane protein 189, TMEM189) inhibited the expression of FAR1 and subsequent ferroptosis (78). Cui had characterized the inhibitory effect of TMEM189 on ferroptosis in different cancer cells. In contrast, Zou claimed that TMEM189 was not involved in ferroptosis regulation. The two contrary results may be explained by different cancer cell lines used in the experiment that expressed discrepant levels of TMEM189. This indicates that the level of TMEM189 protein in different cancer cells results in differences in the regulatory network between cancer cells. Further studies are needed to determine whether the differences in TMEM189 protein levels among varying cancer cell lines are regulated at the gene level, transcription level, or post-translational modification level, which may also contribute to elucidating the role of TMEM189 in ferroptosis.

\section{UPS}

The ubiquitin-proteasome system (UPS) is closely linked to ferroptosis through targeting protein for degradation. The tumor suppressor BRCA1-associated protein 1 (BAP1), a member of the UCH family of deubiquitinase, is negatively associated with many tumors (79-81). BAP1 acts on system $\mathrm{Xc}^{-}$and cleaves the monoubiquitin from lysine 119 of histone 2A (H2AK119Ub) in the SLC7A11 gene, thereby inhibiting the transcription of SLC7A11 and resulting in reduced cystine transport, decreased GSH production, lipid peroxide accumulation, and ultimately promoting the occurrence of ferroptosis $(82,83)$. Further research has demonstrated that the polycomb repressive complex 1 (PRC1), as the main E3 ubiquitin ligase of $\mathrm{H} 2 \mathrm{AUb}$, can enhance the binding of H2AUb to the SLC7A11 promoter and synergistically inhibit the expression of SLC7A11 with BAP1 (84). In addition, mono-ubiquitination of lysine 120 of histone $2 \mathrm{~B}$ (H2BK120Ub) activates SLC7A11 expression, while p53 reduces H2BK120Ub level via promoting nuclear translocation of the deubiquitinase USP7, and finally promoting the occurrence of ferroptosis (85). Studies have shown that SLC7A11 is also positively regulated by deubiquitinase, and OTUB1, a deubiquitinase, can interact directly with SLC7A11 and repress ferroptosis by stabilizing SLC7A11. When the OTUB1 was inactivated, SLC7A11 levels diminished, and ferroptosis was promoted. It was also found that the cluster of differentiation-44 (CD44) could enhance the interaction of OTUB1 with SLC7A11 and prevent ferroptosis (86).

It has been demonstrated that deubiquitinase USP 35 is overexpressed in human lung cancer tissues and cell lines. 
Meanwhile, knockdown of USP35 can promote the degradation of FPN in lung cancer cells and reduce iron exportability that makes cancer cells sensible to ferroptosis, enhancing the chemotherapy effect on lung cancer cells (87). USP11 can stabilize NRF2 by deubiquitination, and USP11 inactivation can promote NRF2 degradation, making cells prone to ferroptosis and reproduction repressed (Figure 4) (88).

\section{AMPK}

AMPK is an AMP-dependent protein kinase that regulates the generation and consumption of ATP to maintain intracellular energy homeostasis. Under energy stress, AMPK is activated and inhibits the physiological processes that consume energy directly or indirectly. Gan et al. recently identified a new mechanism on ferroptosis inhibition in which under energy-deficient conditions, activation of AMPK mediated phosphorylation of acetyl-CoA carboxylase (ACC) and ACC inactivation inhibited the conversion of acetyl-CoA to malonyl-CoA that can generate unsaturated fatty acids and participate in lipid peroxidation. Inhibiting the synthesis of unsaturated fatty acids would ultimately prevent ferroptosis (89-91). Beclin1, the first autophagy-associated protein discovered in mammals, acts as the core of the class III phosphatidylinositol 3-kinase (PtdIns3K) complex to promote the nucleation of autophagosomes (92). Song et al. found that Beclin1 induced ferroptosis independently of the PtdIns3K complex and could form Beclin1-SLC7A11 complex when phosphorylated at Ser90/93/96 by AMPK. This process directly inhibited the cystine transport function of system $\mathrm{Xc}^{-}$, leading to the accumulation of lipid peroxidation and ferroptosis increase $(91,93,94)$. Overexpression of Beclin1 in cancer cells promoted the effect of system $\mathrm{Xc}^{-}$inhibitors on ferroptosis and also promoted the effect of ferroptosis on anticancer in vivo (94). To sum up, AMPK has positive and negative regulatory roles in ferroptosis, so better understanding and accurately judging of the roles of AMPK may be of great but the far-reaching significance for target ferroptosis to treat tumors.

\section{ROLE OF FERROPTOSIS IN HEMATOLOGIC MALIGNANCIES TREATMENT}

\section{Leukemia}

Leukemia is a malignant disease arising from the unlimited proliferation of hematopoietic stem cells, as malignant clones

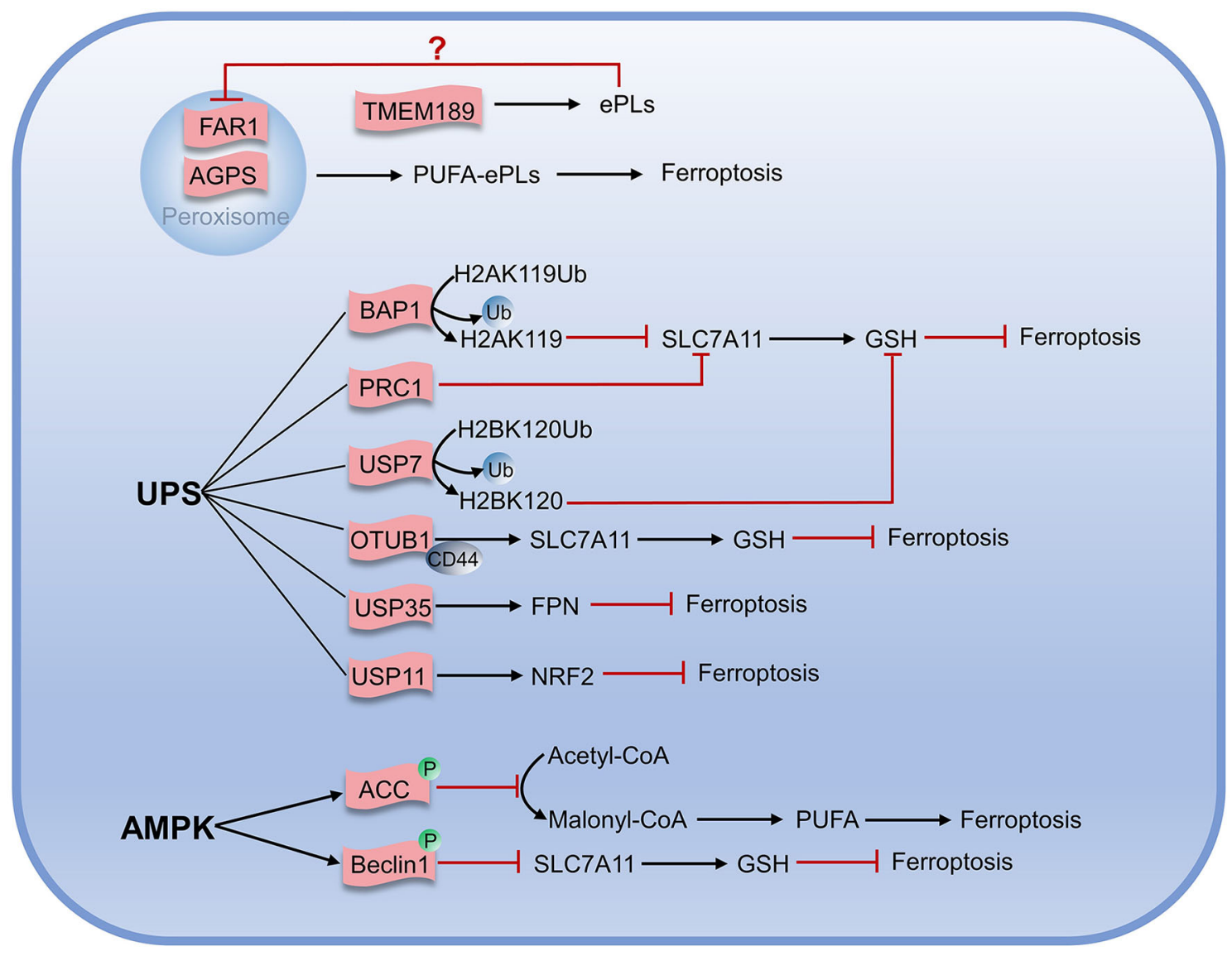

FIGURE 4 | Metabolic regulation associated with ferroptosis. 
of cells could hinder the normal function of hematopoietic cells and affect the development of non-hematopoietic cells. Compared with other tumor cells, leukemia cells display higher transferrin expression and iron content, which causes the accumulation of ROS in leukemia cells more easily and ferroptosis occurs after cells undergo irreparable peroxidative damage. Thereby, promoting ferroptosis via further enhancing the iron content in leukemia cells seems to become a feasible strategy for leukemia treatment, which is also one of the hotspots in this field (95-97).

It has been found that Dihydroartemisinin (DHA), a derivative of the natural drug artemisinin, can induce ferroptosis in acute myeloid leukemia (AML) cells, owing to the capability of activating the phosphorylation of AMPK. Subsequently, AMPK inhibits the mTOR pathway and promotes autophagy, leading to autophagy-dependent degradation of FTH protein and the release of large amounts of free iron, which ultimately induces ferroptosis in AML cells (98). RSL3, as a small molecule inhibitor targeting GPX4, can also trigger a variety of programmed cell deaths, including ferroptosis in AML cells, and enhances the tumor suppressor effect of first-line chemotherapy drugs (cytarabine and adriamycin) on AML cells (99). Similarly, Balasubramanian et al. found that NRF2 may be a new target for the treatment of AML. Using Brusatol, an inhibitor of NRF2, could reduce the ability of NRF2 to eliminate ROS and increase the sensitivity of cytarabine and daunorubicin to AML (100). Ferroptosis induced by iron loading in leukemia cells exert a therapeutic effect, while the occurrence and poor prognosis of leukemia have been identified to be related to intracellular iron accumulation, such as inhibition of red blood cell differentiation when the iron is excessive (101). Therefore, much effort was focused on reducing of the amount of iron in cells. For example, iron chelating agents (DFO and DFX) have been used clinically to reduce the intracellular iron load (102). There are currently relatively few studies on treating leukemia with ferroptosis, and more experiments are required to elucidate the relationship between ferroptosis and leukemia treatment.

\section{Lymphoma}

Diffuse large B-cell lymphoma (DLBCL) is one of the most frequent lymphatic malignancies, with the highest incidence in the non-Hodgkin lymphoma (NHL) family (103, 104). Since Cystine is a crucial negative regulator in the ferroptosis system, the lack of cystine in cells lead to the inhibition of GSH production, which ultimately gives rise to redox imbalance and ferroptosis. Therefore, the inability of lymphocytes to synthesize cystine has been regarded as a breakthrough point for the treatment of lymphoma. At an early stage, Gout et al. revealed that sulfasalazine could be used as a system $\mathrm{Xc}^{-}$inhibitor to significantly reduce the growth of DLBCL in the abdominal cavity of rats (105). Stockwell et al. optimized imidazole-ketoneerastin (IKE) with stronger metabolic stability and water solubility, based on canonical system $\mathrm{Xc}^{-}$inhibitor erastin. IKE could inhibit the growth of DLBCL in mice with more potent therapeutic effects through nanoparticle delivery (106). What's more, Stockwell et al. indicated that inhibition of GPX4 activity also promoted the death of DLBCL cell lines (36). Consistently, GPX4 can reduce lipid peroxides and inhibit ferroptosis, and its overexpression has been demonstrated to be associated with poor prognosis in DLBCL patients (107).

\section{Multiple Myeloma}

Multiple myeloma (MM) is a malignant proliferative disease in plasma cells, accounting for $10 \%$ of hematological malignancies (108). MM is associated with the accumulation of atypical plasma cells in bone marrow, abnormal production of monoclonal immunoglobulin, and increased serum calcium levels (109). GPX4 and SLC7A11 are key regulators of ferroptosis and are highly expressed in MM cells. A novel immunosuppressant Fingolimod (FTY720), can promote ferroptosis by reducing GPX4 and SLC7A11 mRNA and protein levels in U66 cells, enhancing ferroptosis and autophagy through the PP2A/AMPK pathway (110). In MM cells, high proteasome activity is the determinant for degrading misfolded immunoglobulin to ensure expected survival. As a proteasome inhibitor, Bortezomib has been clinically used to treat MM patients (111). However, the autophagy process activated by the accumulation of misfolded immunoglobulin in cells exhibits the resistance of MM against Bortezomib (112). Studies have shown that iron exposure can reduce the activity of the proteasome, hence increasing the efficacy of Bortezomib and Carfilzomib (the second generation of proteasome inhibitors used for MM therapy) in MM cells and leading to severe MM cell death by promoting ferroptosis (113). In order to overcome the drug resistance of Bortezomib, docosahexaenoic acid or eicosapentaenoic acid in combination with Bortezomib were used to enhance the sensitivity of MM cells to Bortezomib (Table 1) (114). Specifically, docosahexaenoic acid and eicosapentaenoic acid can modulate the redox balance in MM cells by reducing the content of GSH in MM cells, thus improving the therapeutic effect of Bortezomib. These combined therapeutic results provide a novel theoretical basis and therapeutic schedule for overcoming MM resistance to Bortezomib and further benefit clinical treatment.

TABLE 1 | Inducers or inhibitors of ferroptosis in hematologic malignancies.

\begin{tabular}{|c|c|c|}
\hline Cancer & Inhibitors/Inducers & Targeted sites \\
\hline \multirow[t]{4}{*}{ Leukemia } & $\mathrm{DHA}$ & AMPK \\
\hline & RSL3 & GPX4 \\
\hline & Brusatol & NRF2 \\
\hline & DFO; DFX & $\mathrm{Fe} 2+$ \\
\hline \multirow[t]{3}{*}{ Lymphoma } & Sulfasalazine & system Xc- \\
\hline & IKE & system Xc- \\
\hline & RSL3 & GPX4 \\
\hline \multirow[t]{3}{*}{$\begin{array}{l}\text { Multiple } \\
\text { myeloma }\end{array}$} & FIY720 & $\begin{array}{l}\text { GPX4; } \\
\text { SLC7A11 }\end{array}$ \\
\hline & Bortezomib; Carfilzomib & Proteasome \\
\hline & $\begin{array}{l}\text { Docosahexaenoic acid; Eicosapentaenoic } \\
\text { acid }\end{array}$ & $\mathrm{GSH}$ \\
\hline
\end{tabular}

acid 
TABLE 2 | Molecular compounds that regulate ferroptosis.

\begin{tabular}{|c|c|c|c|}
\hline & Molecular compound & Targeted sites & Function \\
\hline \multirow[t]{7}{*}{ Inhibitor } & $\alpha$-Tocopherol & PUFA-ROO & Blocks the lipid peroxidation caused by Fenton reaction \\
\hline & Vitamin E & PUFA-ROO & Blocks the lipid peroxidation caused by Fenton reaction \\
\hline & Liproxstatin-1 & PUFA-ROO & Blocks the lipid peroxidation caused by Fenton reaction \\
\hline & Ferrostatin-1 & PUFA-ROO & Blocks the lipid peroxidation caused by Fenton reaction \\
\hline & DFO & $\mathrm{Fe}^{2+}$ & Consumption of iron \\
\hline & DFX & $\mathrm{Fe}^{2+}$ & Consumption of iron \\
\hline & $\mathrm{CoQ}_{10}$ & Lipid peroxidation & Repairs lipid peroxide \\
\hline \multirow[t]{11}{*}{ Inducer } & Erastin & system $\mathrm{Xc}^{-}$ & Prevents cystine from entering cells \\
\hline & IKE & system $\mathrm{Xc}^{-}$ & Prevents cystine from entering cells \\
\hline & Sulfasalazine & system $\mathrm{Xc}^{-}$ & Prevents cystine from entering cells \\
\hline & Sorafenib & system $\mathrm{Xc}^{-}$ & Prevents cystine from entering cells \\
\hline & FIN56 & GPX4 & Induces GPX4 degradation \\
\hline & RSL3 & GPX4 & Covalently inhibits GPX4, leading to accumulation of lipid peroxides \\
\hline & iFSP1 & FSP1 & Consumption of CoQ10 leads to a decrease in GPX4 activity \\
\hline & MTX & DHFR & Inhibits DHFR activity and reduce $\mathrm{BH} 4$ production \\
\hline & Docosahexaenoic acid & GSH & Consumption of GSH \\
\hline & Eicosapentaenoic acid & GSH & Consumption of GSH \\
\hline & Brequinar & $\mathrm{DHODH}$ & Decreases DHODH activity and resultes in accumulation of mitochondrial peroxide lipids \\
\hline
\end{tabular}

\section{CONCLUSION AND PERSPECTIVE}

In recent years, with the deep research on ferroptosis, several pathways other than GPX4-GSH have been identified to modulate lipid peroxidation directly. Meanwhile, large amounts of experiments have also been conducted to elucidate the roles of ferroptosis in hematological malignancies. However, many questions in the ferroptosis field remain to be addressed. For example, to what extent does lipid peroxidation lead to cell rupture and ferroptosis? Are other lipid peroxidation regulatory pathways associated with GPX4-GSH synergistic or merely complementary to GPX4? How can ferroptosis be used as a target of cancer therapy further (In Table 2 we summarize the inhibitors or inducers associated with ferroptosis)? How to avoid the side effects of ferroptosis-related drugs (e.g., the toxic side effects of increased iron intake in leukemia treatment)? In conclusion, as a new modality of regulatory cell death, ferroptosis brings new possibilities for cancer treatment. However, further research is required for elaborating the

\section{REFERENCES}

1. Szabó C. Mechanisms of Cell Necrosis. Crit Care Med (2005) 33(12):4-5. doi: 10.1097/01.CCM.0000187002.88999.CF

2. Padanilam BJ. Cell Death Induced by Acute Renal Injury: A Perspective on the Contributions of Apoptosis and Necrosis. Am J Physiol-Renal Physiol (2003) 284(4):F608-F27. doi: 10.1152/ajprenal.00284.2002

3. Ranganath RM, Rao Nagashree N. Role of Programmed Cell Death in Development. Int Rev Cytol (2001) 202:159-242. Academic Press. doi: 10.1016/S0074-7696(01)02005-8

4. Hirschhorn T, Stockwell BR. The Development of the Concept of Ferroptosis. Free Radical Biol Med (2019) 133:130-43. doi: 10.1016/j.freeradbiomed.2018.09.043

5. Sen S. Programmed Cell Death: Concept, Mechanism and Control. Biol Rev (1992) 67(3):287-319. doi: 10.1111/j.1469-185X.1992.tb00727.x

6. Daneva A, Gao Z, Van Durme M, Nowack MK. Functions and Regulation of Programmed Cell Death in Plant Development. Annu Rev Cell Dev Biol (2016) 32(1):441-68. doi: 10.1146/annurev-cellbio-111315-124915 mechanism of ferroptosis and its implications in cancer, which is of great scientific significance.

\section{AUTHOR CONTRIBUTIONS}

FW and HL conceptualized the study. FW oversaw the literature review involved in all aspects of designing and writing the manuscript. FW and HL performed the literature review. FW, $\mathrm{ZM}$, and HL wrote the manuscript and designed the figures. ZZ, $\mathrm{YG}$, and $\mathrm{ZM}$ provided input on the discussion of various sections. All authors contributed to the article and approved the submitted version.

\section{FUNDING}

This work was supported by the National Natural Science Foundation of China Grants 31770827 and 21736002 to FW, 31870791 and 91753205 to ZM.
7. Barros LF, Hermosilla T, Castro J. Necrotic Volume Increase and the Early Physiology of Necrosis. Comp Biochem Physiol Part A: Mol Integr Physiol (2001) 130(3):401-9. doi: 10.1016/S1095-6433(01)00438-X

8. Dixon SJ, Lemberg KM, Lamprecht MR, Skouta R, Zaitsev EM, Gleason CE, et al. Ferroptosis: An Iron-Dependent Form of Nonapoptotic Cell Death. Cell (2012) 149(5):1060-72. doi: 10.1016/j.cell.2012.03.042

9. Jiang X, Stockwell BR, Conrad M. Ferroptosis: Mechanisms, Biology and Role in Disease. Nat Rev Mol Cell Biol (2021) 22(4):266-82. doi: 10.1038/ s41580-020-00324-8

10. Xie Y, Hou W, Song X, Yu Y, Huang J, Sun X, et al. Ferroptosis: Process and Function. Cell Death Differ (2016) 23(3):369-79. doi: 10.1038/cdd.2015.158

11. Yang WS, Kim KJ, Gaschler MM, Patel M, Shchepinov MS, Stockwell BR. Peroxidation of Polyunsaturated Fatty Acids by Lipoxygenases Drives Ferroptosis. Proc Natl Acad Sci USA (2016) 113(34):E4966-E75. doi: 10.1073/pnas.1603244113

12. D'Herde K, Krysko DV. Oxidized PEs Trigger Death. Nat Chem Biol (2017) 13(1):4-5. doi: 10.1038/nchembio.2261 
13. Kagan VE, Mao G, Qu F, Angeli JPF, Doll S, Croix CS, et al. Oxidized Arachidonic and Adrenic PEs Navigate Cells to Ferroptosis. Nat Chem Biol (2017) 13(1):81-90. doi: 10.1038/nchembio.2238

14. D'Herde K, Krysko DA-O. Ferroptosis: Oxidized PEs Trigger Death. Nat Chem Biol (2017) 13(1):4-5. doi: 10.1038/nchembio.2261

15. Hishikawa D, Shindou H, Kobayashi S, Nakanishi H, Taguchi R, Shimizu T. Discovery of a Lysophospholipid Acyltransferase Family Essential for Membrane Asymmetry and Diversity. Proc Natl Acad Sci USA (2008) 105 (8):2830-5. doi: 10.1073/pnas.0712245105

16. Kuhn H, Banthiya S, van Leyen K. Mammalian Lipoxygenases and Their Biological Relevance. Biochim Biophys Acta (2015) 1851(4):308-30. doi: 10.1016/j.bbalip.2014.10.002

17. Plount Price ML, Jorgensen WL. Analysis of Binding Affinities for Celecoxib Analogues With COX-1 and COX-2 From Combined Docking and Monte Carlo Simulations and Insight Into the COX-2/COX-1 Selectivity. J Am Chem Soc (2000) 122(39):9455-66. doi: 10.1021/ja001018c

18. Brash AR. Lipoxygenases: Occurrence, Functions, Catalysis, and Acquisition of Substrate *. J Biol Chem (1999) 274(34):23679-82. doi: 10.1074/ jbc. 274.34 .23679

19. Shah R, Shchepinov MS, Pratt DA. Resolving the Role of Lipoxygenases in the Initiation and Execution of Ferroptosis. ACS Cent Sci (2018) 4(3):38796. doi: 10.1021/acscentsci.7b00589

20. Clemente SM, Martínez-Costa OH, Monsalve M, Samhan-Arias AK. Targeting Lipid Peroxidation for Cancer Treatment. Molecules (2020) 25 (21):5144. doi: 10.3390/molecules25215144

21. Gaschler MM, Stockwell BR. Lipid Peroxidation in Cell Death. Biochem Biophys Res Commun (2017) 482(3):419-25. doi: 10.1016/j.bbrc.2016.10.086

22. Hassannia B, Vandenabeele P, Vanden Berghe T. Targeting Ferroptosis to Iron Out Cancer. Cancer Cell (2019) 35(6):830-49. doi: 10.1016/j.ccell. 2019.04.002

23. Stockwell BR, Friedmann Angeli JP, Bayir H, Bush AI, Conrad M, Dixon SJ, et al. Ferroptosis: A Regulated Cell Death Nexus Linking Metabolism, Redox Biology, and Disease. Cell (2017) 171(2):273-85. doi: 10.1016/j.cell. 2017.09.021

24. Kagan VE, Tyurina YY, Sun WY, Vlasova II, Dar H, Tyurin VA, et al. Redox Phospholipidomics of Enzymatically Generated Oxygenated Phospholipids as Specific Signals of Programmed Cell Death. Free Radical Biol Med (2020) 147:231-41. doi: 10.1016/j.freeradbiomed.2019.12.028

25. Traber MG, Atkinson J. Vitamin E, Antioxidant and Nothing More. Free Radic Biol Med (2007) 43(1):4-15. doi: 10.1016/j.freeradbiomed.2007.03.024

26. Kajarabille N, Latunde-Dada GO. Programmed Cell-Death by Ferroptosis: Antioxidants as Mitigators. Int J Mol Sci (2019) 20(19):4968. doi: 10.3390/ ijms 20194968

27. Friedmann Angeli JP, Schneider M, Proneth B, Tyurina YY, Tyurin VA, Hammond VJ, et al. Inactivation of the Ferroptosis Regulator Gpx4 Triggers Acute Renal Failure in Mice. Nat Cell Biol (2014) 16(12):1180-91. doi: $10.1038 /$ ncb3064

28. Shah R, Margison K, Pratt DA. The Potency of Diarylamine RadicalTrapping Antioxidants as Inhibitors of Ferroptosis Underscores the Role of Autoxidation in the Mechanism of Cell Death. ACS Chem Biol (2017) 12 (10):2538-45. doi: 10.1021/acschembio.7b00730

29. Koppula P, Zhuang L, Gan B. Cytochrome P450 Reductase (POR) as a Ferroptosis Fuel. Protein Cell (2021) 12(9):675-9. doi: 10.1007/s13238-02100823-0

30. Yan B, Ai Y, Sun Q, Ma Y, Cao Y, Wang J, et al. Membrane Damage During Ferroptosis Is Caused by Oxidation of Phospholipids Catalyzed by the Oxidoreductases POR and CYB5R1. Mol Cell (2021) 81(2):355-69.e10. doi: 10.1016/j.molcel.2020.11.024

31. Bersuker K, Hendricks JM, Li Z, Magtanong L, Ford B, Tang PH, et al. The CoQ Oxidoreductase FSP1 Acts Parallel to GPX4 to Inhibit Ferroptosis. Nature (2019) 575(7784):688-92. doi: 10.1038/s41586-019-1705-2

32. Seibt TM, Proneth B, Conrad M. Role of GPX4 in Ferroptosis and Its Pharmacological Implication. Free Radical Biol Med (2019) 133:144-52. doi: 10.1016/j.freeradbiomed.2018.09.014

33. Conrad M, Sato H. The Oxidative Stress-Inducible Cystine/Glutamate Antiporter, System X (C) (-) : Cystine Supplier and Beyond. Amino Acids (2012) 42(1):231-46. doi: 10.1007/s00726-011-0867-5
34. Meister A. [3] Glutathione Biosynthesis and Its Inhibition. Methods Enzymol (1995) 252:26-30. Academic Press. doi: 10.1016/0076-6879(95)52005-8

35. Lu SC. Glutathione Synthesis. Biochim Biophys Acta (2013) 1830(5):314353. doi: 10.1016/j.bbagen.2012.09.008

36. Yang Wan S, SriRamaratnam R, Welsch Matthew E, Shimada K, Skouta R, Viswanathan Vasanthi S, et al. Regulation of Ferroptotic Cancer Cell Death by GPX4. Cell (2014) 156(1):317-31.

37. Yi R, Wang H, Deng C, Wang X, Yao L, Niu W, et al. Dihydroartemisinin Initiates Ferroptosis in Glioblastoma Through GPX4 Inhibition. Biosci Rep (2020) 40(6):BSR20193314. doi: 10.1042/BSR20193314

38. Hu Q, Zhang Y, Lou H, Ou Z, Liu J, Duan W, et al. GPX4 and Vitamin E Cooperatively Protect Hematopoietic Stem and Progenitor Cells From Lipid Peroxidation and Ferroptosis. Cell Death Dis (2021) 12(7):706-. doi: 10.1038/s41419-021-04008-9

39. Carlson BA, Tobe R, Yefremova E, Tsuji PA, Hoffmann VJ, Schweizer U, et al. Glutathione Peroxidase 4 and Vitamin E Cooperatively Prevent Hepatocellular Degeneration. Redox Biol (2016) 9:22-31. doi: 10.1016/ j.redox.2016.05.003

40. Wu M, Xu L-G, Li X, Zhai Z, Shu H-B. AMID, an Apoptosis-Inducing Factor-Homologous Mitochondrion-Associated Protein, Induces CaspaseIndependent Apoptosis *. J Biol Chem (2002) 277(28):25617-23. doi: 10.1074/jbc.M202285200

41. Ohiro Y, Garkavtsev I, Kobayashi S, Sreekumar KR, Nantz R, Higashikubo BT, et al. A Novel P53-Inducible Apoptogenic Gene, PRG3, Encodes a Homologue of the Apoptosis-Inducing Factor (AIF). FEBS Lett (2002) 524 (1-3):163-71. doi: 10.1016/S0014-5793(02)03049-1

42. Elguindy MM, Nakamaru-Ogiso E. Apoptosis-Inducing Factor (AIF) and Its Family Member Protein, AMID, Are Rotenone-Sensitive NADH: Ubiquinone Oxidoreductases (NDH-2). J Biol Chem (2015) 290 (34):20815-26. doi: 10.1074/jbc.M115.641498

43. Doll S, Freitas FP, Shah R, Aldrovandi M, da Silva MC, Ingold I, et al. FSP1 Is a Glutathione-Independent Ferroptosis Suppressor. Nature (2019) 575 (7784):693-8. doi: 10.1038/s41586-019-1707-0

44. Dai E, Zhang W, Cong D, Kang R, Wang J, Tang D. AIFM2 Blocks Ferroptosis Independent of Ubiquinol Metabolism. Biochem Biophys Res Commun (2020) 523(4):966-71. doi: 10.1016/j.bbrc.2020.01.066

45. Mei J, Webb S, Zhang B, Shu HB. The P53-Inducible Apoptotic Protein AMID Is Not Required for Normal Development and Tumor Suppression. Oncogene (2006) 25(6):849-56. doi: 10.1038/sj.onc.1209121

46. Ebenhoch R, Bauer M, Reinert D, Kersting A, Huber S, Schmid A, et al. Biophysical and Structural Investigation of the Regulation of Human GTP Cyclohydrolase I by Its Regulatory Protein GFRP. J Struct Biol (2021) 213 (1):107691. doi: 10.1016/j.jsb.2020.107691

47. Kraft VAN, Bezjian CT, Pfeiffer S, Ringelstetter L, Müller C, Zandkarimi F, et al. GTP Cyclohydrolase 1/Tetrahydrobiopterin Counteract Ferroptosis Through Lipid Remodeling. ACS Cent Sci (2020) 6(1):41-53. doi: 10.1021/acscentsci.9b01063

48. Soula M, Weber RA, Zilka O, Alwaseem H, La K, Yen F, et al. Metabolic Determinants of Cancer Cell Sensitivity to Canonical Ferroptosis Inducers. Nat Chem Biol (2020) 16(12):1351-60. doi: 10.1038/s41589-020-0613-y

49. Crabtree MJ, Channon KM. Synthesis and Recycling of Tetrahydrobiopterin in Endothelial Function and Vascular Disease. Nitric Oxide (2011) 25(2):818. doi: 10.1016/j.niox.2011.04.004

50. Sun W-Y, Tyurin VA, Mikulska-Ruminska K, Shrivastava IH, Anthonymuthu TS, Zhai Y-J, et al. Phospholipase Ipla2 $\beta$ Averts Ferroptosis by Eliminating a Redox Lipid Death Signal. Nat Chem Biol (2021) 17(4):465-76. doi: 10.1038/s41589-020-00734-x

51. Chen D, Chu B, Yang X, Liu Z, Jin Y, Kon N, et al. Ipla2ß-Mediated Lipid Detoxification Controls P53-Driven Ferroptosis Independent of GPX4. Nat Commun (2021) 12(1):3644-. doi: 10.1038/s41467-021-23902-6

52. Mao C, Liu X, Zhang Y, Lei G, Yan Y, Lee H, et al. DHODH-Mediated Ferroptosis Defence Is a Targetable Vulnerability in Cancer. Nature (2021) 593(7860):586-90. doi: 10.1038/s41586-021-03539-7

53. Chen Y, Fan Z, Yang Y, Gu C. Iron Metabolism and Its Contribution to Cancer (Review). Int J Oncol (2019) 54(4):1143-54. doi: 10.3892/ijo.2019.4720

54. Li Y, Huang X, Wang J, Huang R, Wan D. Regulation of Iron Homeostasis and Related Diseases. Mediators Inflamm (2020) 2020:6062094-. doi: $10.1155 / 2020 / 6062094$ 
55. Li J, Cao F, Yin H-l, Huang Z-j, Lin Z-t, Mao N, et al. Ferroptosis: Past, Present and Future. Cell Death Dis (2020) 11(2):88. doi: 10.1038/s41419020-2298-2

56. Stockwell BR, Jiang X. The Chemistry and Biology of Ferroptosis. Cell Chem Biol (2020) 27(4):365-75. doi: 10.1016/j.chembiol.2020.03.013

57. Fleming MD, Romano MA, Su MA, Garrick LM, Garrick MD, Andrews NC. Nramp2 Is Mutated in the Anemic Belgrade (B) Rat: Evidence of a Role for Nramp2 in Endosomal Iron Transport. Proc Natl Acad Sci USA (1998) 95 (3):1148-53. doi: 10.1073/pnas.95.3.1148

58. Fuqua BK, Lu Y, Darshan D, Frazer DM, Wilkins SJ, Wolkow N, et al. The Multicopper Ferroxidase Hephaestin Enhances Intestinal Iron Absorption in Mice. PloS One (2014) 9(6):e98792-e. doi: 10.1371/journal.pone.0098792

59. Frazer DM, Anderson GJ. The Regulation of Iron Transport. BioFactors (2014) 40(2):206-14. doi: 10.1002/biof.1148

60. Torti SV, Torti FM. Iron and Cancer: More Ore to be Mined. Nat Rev Cancer (2013) 13(5):342-55. doi: 10.1038/nrc3495

61. Park E, Chung SW. ROS-Mediated Autophagy Increases Intracellular Iron Levels and Ferroptosis by Ferritin and Transferrin Receptor Regulation. Cell Death Dis (2019) 10(11):822-. doi: 10.1038/s41419-019-2064-5

62. Tang L-J, Zhou Y-J, Xiong X-M, Li N-S, Zhang J-J, Luo X-J, et al. UbiquitinSpecific Protease 7 Promotes Ferroptosis via Activation of the P53/TfR1 Pathway in the Rat Hearts After Ischemia/Reperfusion. Free Radical Biol Med (2021) 162:339-52. doi: 10.1016/j.freeradbiomed.2020.10.307

63. Geng N, Shi BJ, Li SL, Zhong ZY, Li YC, Xua WL, et al. Knockdown of Ferroportin Accelerates Erastin-Induced Ferroptosis in Neuroblastoma Cells. Eur Rev Med Pharmacol Sci (2018) 22(12):3826-36. doi: 10.26355/eurrev_201806_15267

64. Hao L, Mi J, Song L, Guo Y, Li Y, Yin Y, et al. SLC40A1 Mediates Ferroptosis and Cognitive Dysfunction in Type 1 Diabetes. Neuroscience (2021) 463:216-26. doi: 10.1016/j.neuroscience.2021.03.009

65. Theil EC. Ferritin Protein Nanocages-the Story. Nanotechnol Percept (2012) 8(1):7-16. doi: 10.4024/N03TH12A.ntp.08.01

66. Tosha T, Behera RK, Ng H-L, Bhattasali O, Alber T, Theil EC. Ferritin Protein Nanocage Ion Channels: Gating by N-Terminal Extensions. J Biol Chem (2012) 287(16):13016-25. doi: 10.1074/jbc.M111.332734

67. Gryzik M, Asperti M, Denardo A, Arosio P, Poli M. NCOA4-Mediated Ferritinophagy Promotes Ferroptosis Induced by Erastin, But Not by RSL3 in HeLa Cells. Biochim Biophys Acta (BBA) - Mol Cell Res (2021) 1868 (2):118913. doi: 10.1016/j.bbamcr.2020.118913

68. Kerins MJ, Ooi A. The Roles of NRF2 in Modulating Cellular Iron Homeostasis. Antioxidants Redox Signaling (2018) 29(17):1756-73. doi: 10.1089/ars.2017.7176

69. Rouault TA. The Role of Iron Regulatory Proteins in Mammalian Iron Homeostasis and Disease. Nat Chem Biol (2006) 2(8):406-14. doi: 10.1038/ nchembio807

70. Hernández-Gallardo AK, Missirlis F. Cellular Iron Sensing and Regulation: Nuclear IRP1 Extends a Classic Paradigm. Biochim Biophys Acta (BBA) Mol Cell Res (2020) 1867(7):118705.

71. Meyron-Holtz EG, Ghosh MC, Iwai K, LaVaute T, Brazzolotto X, Berger UV, et al. Genetic Ablations of Iron Regulatory Proteins 1 and 2 Reveal Why Iron Regulatory Protein 2 Dominates Iron Homeostasis. EMBO J (2004) 23 (2):386-95. doi: 10.1038/sj.emboj.7600041

72. Cairo G, Recalcati S, Pietrangelo A, Minotti G. The Iron Regulatory Proteins: Targets and Modulators of Free Radical Reactions and Oxidative. Free Radic Biol Med (2002) 32(12):1237-43. doi: 10.1016/ s0891-5849(02)00825-0

73. Popovic Z, Templeton Douglas M. Interaction of Iron Regulatory Protein-1 (IRP-1) With ATP/ADP Maintains a non-IRE-Binding State. Biochem J (2010) 430(2):315-24. doi: 10.1042/BJ20100111

74. Dean JM, Lodhi IJ. Structural and Functional Roles of Ether Lipids. Protein Cell (2018) 9(2):196-206. doi: 10.1007/s13238-017-0423-5

75. Lee H, Zhuang L, Gan B. Ether Phospholipids Govern Ferroptosis. J Genet Genomics (2021) 48(7):517-9. doi: 10.1016/j.jgg.2021.05.003

76. Zou Y, Henry WS, Ricq EL, Graham ET, Phadnis VV, Maretich P, et al. Plasticity of Ether Lipids Promotes Ferroptosis Susceptibility and Evasion. Nature (2020) 585(7826):603-8. doi: 10.1038/s41586-020-2732-8

77. Cui W, Liu D, Gu W, Chu B. Peroxisome-Driven Ether-Linked Phospholipids Biosynthesis Is Essential for Ferroptosis. Cell Death Differ (2021) 28(8):2536-51. doi: 10.1038/s41418-021-00769-0
78. Werner ER, Keller MA, Sailer S, Lackner K, Koch J, Hermann M, et al. The TMEM189 Gene Encodes Plasmanylethanolamine Desaturase Which Introduces the Characteristic Vinyl Ether Double Bond Into Plasmalogens. Proc Natl Acad Sci USA (2020) 117(14):7792-8. doi: 10.1073/ pnas. 1917461117

79. Asada S, Goyama S, Inoue D, Shikata S, Takeda R, Fukushima T, et al. Mutant ASXL1 Cooperates With BAP1 to Promote Myeloid Leukaemogenesis. Nat Commun (2018) 9(1):2733. doi: 10.1038/s41467018-05085-9

80. Dey A, Seshasayee D, Noubade R, French DM, Liu J, Chaurushiya MS, et al. Loss of the Tumor Suppressor BAP1 Causes Myeloid Transformation. Science (2012) 337(6101):1541-6. doi: 10.1126/science.1221711

81. Bai J, Chen Z, Chen C, Zhang M, Zhang Y, Song J, et al. Reducing Hyperactivated BAP1 Attenuates Mutant ASXL1-Driven Myeloid Malignancies in Human Haematopoietic Cells. Cancer Lett (2021) 519:7890. doi: 10.1016/j.canlet.2021.06.019

82. Zhang Y, Shi J, Liu X, Feng L, Gong Z, Koppula P, et al. BAP1 Links Metabolic Regulation of Ferroptosis to Tumour Suppression. Nat Cell Biol (2018) 20(10):1181-92. doi: 10.1038/s41556-018-0178-0

83. Zhang Y, Zhuang L, Gan B. BAP1 Suppresses Tumor Development by Inducing Ferroptosis Upon SLC7A11 Repression. Mol Cell Oncol (2018) 6 (1):1536845-. doi: 10.1080/23723556.2018.1536845

84. Zhang Y, Koppula P, Gan B. Regulation of H2A Ubiquitination and SLC7A11 Expression by BAP1 and PRC1. Cell Cycle (2019) 18(8):773-83. doi: 10.1080/15384101.2019.1597506

85. Wang Y, Yang L, Zhang X, Cui W, Liu Y, Sun Q-R, et al. Epigenetic Regulation of Ferroptosis by H2B Monoubiquitination and P53. EMBO Rep (2019) 20(7):e47563-e. doi: 10.15252/embr.201847563

86. Liu T, Jiang L, Tavana O, Gu W. The Deubiquitylase OTUB1 Mediates Ferroptosis via Stabilization of SLC7A11. Cancer Res (2019) 79(8):1913-24. doi: 10.1158/0008-5472.CAN-18-3037

87. Tang Z, Jiang W, Mao M, Zhao J, Chen J, Cheng N. Deubiquitinase USP35 Modulates Ferroptosis in Lung Cancer via Targeting Ferroportin. Clin Transl Med (2021) 11(4):e390-e. doi: 10.1002/ctm2.390

88. Meng C, Zhan J, Chen D, Shao G, Zhang H, Gu W, et al. The Deubiquitinase USP11 Regulates Cell Proliferation and Ferroptotic Cell Death via Stabilization of NRF2 USP11 Deubiquitinates and Stabilizes NRF2. Oncogene (2021) 40(9):1706-20. doi: 10.1038/s41388-021-01660-5

89. Lee H, Zandkarimi F, Zhang Y, Meena JK, Kim J, Zhuang L, et al. EnergyStress-Mediated AMPK Activation Inhibits Ferroptosis. Nat Cell Biol (2020) 22(2):225-34. doi: 10.1038/s41556-020-0461-8

90. Lee H, Zhuang L, Gan B. Energy Stress Inhibits Ferroptosis via AMPK. Mol Cell Oncol (2020) 7(4):1761242-. doi: 10.1080/23723556.2020.1761242

91. Wei X, Yi X, Zhu X-H, Jiang D-S. Posttranslational Modifications in Ferroptosis. Oxid Med Cell Longev (2020) 2020:8832043-. doi: 10.1155/ 2020/8832043

92. Funderburk SF, Wang QJ, Yue Z. The Beclin 1-VPS34 Complex-at the Crossroads of Autophagy and Beyond. Trends Cell Biol (2010) 20(6):355-62. doi: $10.1016 /$ j.tcb.2010.03.002

93. Kang R, Zhu S, Zeh HJ, Klionsky DJ, Tang D. BECN1 Is a New Driver of Ferroptosis. Autophagy (2018) 14(12):2173-5. doi: 10.1080/15548627.2018. 1513758

94. Song X, Zhu S, Chen P, Hou W, Wen Q, Liu J, et al. AMPK-Mediated BECN1 Phosphorylation Promotes Ferroptosis by Directly Blocking System X(c)(-) Activity. Curr Biol (2018) 28(15):2388-99.e5. doi: 10.1016/ j.cub.2018.05.094

95. Grignano E, Birsen R, Chapuis N, Bouscary D. From Iron Chelation to Overload as a Therapeutic Strategy to Induce Ferroptosis in Leukemic Cells. Front Oncol (2020) 10:1859. doi: 10.3389/fonc.2020.586530

96. Wang F, Lv H, Zhao B, Zhou L, Wang S, Luo J, et al. Iron and Leukemia: New Insights for Future Treatments. J Exp Clin Cancer Res (2019) 38 (1):406-. doi: 10.1186/s13046-019-1397-3

97. Hunter RL, Bennett B, Towns M, Vogler WR. Transferrin in Disease II: Defects in the Regulation of Transferrin Saturation With Iron Contribute to Susceptibility to Infection. Am J Clin Pathol (1984) 81(6):748-53. doi: 10.1093/ajcp/81.6.748

98. Du J, Wang T, Li Y, Zhou Y, Wang X, Yu X, et al. DHA Inhibits Proliferation and Induces Ferroptosis of Leukemia Cells Through Autophagy Dependent 
Degradation of Ferritin. Free Radical Biol Med (2019) 131:356-69. doi: 10.1016/j.freeradbiomed.2018.12.011

99. Yu Y, Xie Y, Cao L, Yang L, Yang M, Lotze MT, et al. The Ferroptosis Inducer Erastin Enhances Sensitivity of Acute Myeloid Leukemia Cells to Chemotherapeutic Agents. Mol Cell Oncol (2015) 2(4):e1054549-e. doi: 10.1080/23723556.2015.1054549

100. Karathedath S, Rajamani BM, Musheer Aalam SM, Abraham A, Varatharajan S, Krishnamurthy P, et al. Role of NF-E2 Related Factor 2 (Nrf2) on Chemotherapy Resistance in Acute Myeloid Leukemia (AML) and the Effect of Pharmacological Inhibition of Nrf2. PloS One (2017) 12(5): e0177227-e. doi: 10.1371/journal.pone.0177227

101. Taoka K, Kumano K, Nakamura F, Hosoi M, Goyama S, Imai Y, et al. The Effect of Iron Overload and Chelation on Erythroid Differentiation. Int $J$ Hematol (2012) 95(2):149-59. doi: 10.1007/s12185-011-0988-3

102. Neufeld EJ. Oral Chelators Deferasirox and Deferiprone for Transfusional Iron Overload in Thalassemia Major: New Data, New Questions. Blood (2006) 107(9):3436-41. doi: 10.1182/blood-2006-02-002394

103. Tilly H, Gomes da Silva M, Vitolo U, Jack A, Meignan M, Lopez-Guillermo A, et al. Diffuse Large B-Cell Lymphoma (DLBCL): ESMO Clinical Practice Guidelines for Diagnosis, Treatment and Follow-Up. Ann Oncol (2015) 26 (Suppl 5):v116-25. doi: 10.1093/annonc/mdv304

104. Martelli M, Ferreri AJ, Agostinelli C, Di Rocco A, Pfreundschuh M, Pileri SA. Diffuse Large B-Cell Lymphoma. Crit Rev Oncol Hematol (2013) 87(2):14671. doi: 10.1016/j.critrevonc.2012.12.009

105. Gout PW, Buckley AR, Simms CR, Bruchovsky N. Sulfasalazine, a Potent Suppressor of Lymphoma Growth by Inhibition of the Xc- Cystine Transporter: A New Action for an Old Drug. Leukemia (2001) 15 (10):1633-40. doi: 10.1038/sj.leu.2402238

106. Zhang Y, Tan H, Daniels JD, Zandkarimi F, Liu H, Brown LM, et al. Imidazole Ketone Erastin Induces Ferroptosis and Slows Tumor Growth in a Mouse Lymphoma Model. Cell Chem Biol (2019) 26(5):623-33.e9. doi: 10.1016/j.chembiol.2019.01.008

107. Kinowaki Y, Kurata M, Ishibashi S, Ikeda M, Tatsuzawa A, Yamamoto M, et al. Glutathione Peroxidase 4 Overexpression Inhibits ROS-Induced Cell Death in Diffuse Large B-Cell Lymphoma. Lab Invest (2018) 98(5):609-19. doi: 10.1038/s41374-017-0008-1

108. Kyle RA, Rajkumar SV. Multiple Myeloma. Blood (2008) 111(6):2962-72. doi: 10.1182/blood-2007-10-078022
109. Joshua DE, Bryant C, Dix C, Gibson J, Ho J. Biology and Therapy of Multiple Myeloma. Med J Aust (2019) 210(8):375-80. doi: 10.5694/mja2.50129

110. Zhong Y, Tian F, Ma H, Wang H, Yang W, Liu Z, et al. FTY720 Induces Ferroptosis and Autophagy via PP2A/AMPK Pathway in Multiple Myeloma Cells. Life Sci (2020) 260:118077. doi: 10.1016/j.lfs.2020.118077

111. Meister S, Schubert U, Neubert K, Herrmann K, Burger R, Gramatzki M, et al. Extensive Immunoglobulin Production Sensitizes Myeloma Cells for Proteasome Inhibition. Cancer Res (2007) 67(4):1783-92. doi: 10.1158/00085472.CAN-06-2258

112. Cengiz Seval G, Beksac M. The Safety of Bortezomib for the Treatment of Multiple Myeloma. Expert Opin Drug Saf (2018) 17(9):953-62. doi: 10.1080/ 14740338.2018.1513487

113. Bordini J, Morisi F, Cerruti F, Cascio P, Camaschella C, Ghia P, et al. Iron Causes Lipid Oxidation and Inhibits Proteasome Function in Multiple Myeloma Cells: A Proof of Concept for Novel Combination Therapies. Cancers (Basel) (2020) 12(4):970. doi: 10.3390/cancers12040970

114. Chen J, Zaal EA, Berkers CR, Ruijtenbeek R, Garssen J, Redegeld FA. Omega3 Fatty Acids DHA and EPA Reduce Bortezomib Resistance in Multiple Myeloma Cells by Promoting Glutathione Degradation. Cells (2021) 10 (9):2287. doi: 10.3390/cells10092287

Conflict of Interest: The authors declare that the research was conducted in the absence of any commercial or financial relationships that could be construed as a potential conflict of interest.

Publisher's Note: All claims expressed in this article are solely those of the authors and do not necessarily represent those of their affiliated organizations, or those of the publisher, the editors and the reviewers. Any product that may be evaluated in this article, or claim that may be made by its manufacturer, is not guaranteed or endorsed by the publisher.

Copyright $\odot 2022$ Lan, Gao, Zhao, Mei and Wang. This is an open-access article distributed under the terms of the Creative Commons Attribution License (CC BY). The use, distribution or reproduction in other forums is permitted, provided the original author(s) and the copyright owner(s) are credited and that the original publication in this journal is cited, in accordance with accepted academic practice. No use, distribution or reproduction is permitted which does not comply with these terms. 